Revista do Programa de Pós-Graduação em Mídia e Cotidiano

Artigo Seção Dossiê Mídia e Cidade

Número 6. Volume 6 /Julho 2015

(C) 2015 by UFF

\title{
IMEDIAPOLIS: CIDADE, TECNOLOGIA E POLÍTICA
}

\section{IMEDIAPOLIS: CITY, TECHNOLOGY AND POLITICS}

\author{
Guilherme Fráguas NOBRE ${ }^{2}$
}

Resumo: Na Parte I o artigo busca as diferentes definições que têm sido associadas ao termo mediapolis, procurando relacioná-las aos conceitos de cidade, tecnologia e política. A pesquisa registrou o uso do termo mediapolis no contexto teórico da arquitetura, das comunicações, da sociologia e da política, bem como no da filosofia. $\mathrm{Na}$ Parte II o artigo apresenta os agentes sociáveis artificiais, mídias autônomas, inteligentes e capazes de interação/cooperação com seres humanos. Esses novos atores sociais subvertem os conceitos de cidade e de cidadão, sugerindo a evolução da Mediapolis para iMediapolis - intelligent media polis (cidade de mídias inteligentes). Conclui-se com a necessidade de rever as contribuições de Silverstone e demais autores sob a luz dos atores sociais artificiais.

Palavras-chave: iMediapolis; Cidade; Tecnologia; Política; Agentes Sociais Artificiais;

Abstract: At its Part I the article searches for the different definitions that have been linked to the term Mediapolis, trying to relate them with the concepts of city, technology and politics. The survey has registered the use of Mediapolis in the theoretical contexts of architecture, communications, sociology, politics, and philosophy. At its Part II the article presents the artificial social agents, media that is independent, intelligent and capable of interaction/cooperation with humans. These new social actors subvert the concepts of city and citizenship, suggesting the evolution of Mediapolis to the new concept of iMediapolis - intelligent media polis. The article concludes with the need to review the contributions from Silverstone and the other authors, given the presence of the artificial social actors.

Keywords: iMediapolis, city, technology, politics, artificial social agents.

\footnotetext{
1 A Parte I do trabalho foi apresentada no XI Simpósio Internacional de Artemídia e Cultura Digital, Lisboa e Coimbra, 2014. A Parte II foi apresentada no $8^{\circ}$ LUSOCOM, Lisboa, Universidade Lusófona, 2009.

${ }^{2}$ Economista pela UFMG, mestre em economia pela UFPR, doutor em comunicação pela USP, pós doutor em comunicação pela Université Stendhal, visiting scholar na McMaster University, pós doutorando em comunicação na ECA/USP.
} 


\section{míDiA

\section{INTRODUÇÃO}

O conceito de mediapolis (media + polis) evoca outros neologismos, tais como biopolis, fusionopolis (Business Times, 2009) e technopolis (Inkinen, 2004). Para além do óbvio, na tradução quase literal de cidade da mídia ou cidade de mídia, a verdade é que mediapolis implica uma leitura que ultrapassa a cidade e a mídia. Um dos objetivos deste artigo é sair em busca das definições e usos deste conceito, de modo a coligir e comparar as visões de diversos autores que trabalharam com este tema; para, quem sabe, proceder a uma arqueologia ou geologia do conceito.

Mediapolis pode, sim, ser uma cidade da mídia (um parque industrial para empresas de tecnologia) e uma cidade de mídia (um ambiente virtual que emula a cidade real). Contudo, a relação entre a cidade e a tecnologia vai além, envolvendo a arquitetura pública de prédios e a "arquitetura" de ambientes públicos de interação mediada. A mídia enquanto parte da (i)materialização da esfera pública, um "espaço para a ação e participação pública" (Tufte, 2011), eleva a mediapolis a conceito central da comunicação política - sugerindo uma releitura da democracia e da cidadania à sua luz. Contudo, é Silverstone (2006) que propõe um salto qualitativo, sugerindo a mediapolis como uma instância ética e moral da visibilidade pública.

Outro objetivo do artigo é mostrar como os conceitos de cidade e de cidadão (assim como os de cidadania e democracia) tornam-se confusos com a introdução de máquinas autônomas e inteligentes, mídias capazes de sociabilização e de cooperação com seres humanos. O texto está divido em três partes: os conceitos de mediapolis são coligidos na Parte I, enquanto que os atores sociais artificiais são abordados na Parte II. A Parte III vai relacionar os conceitos das duas anteriores, e é seguida da conclusão. 


\section{Parte I}

\section{O conceito de Mediapolis}

Vários autores têm usado o conceito de "Mediapolis" em contextos mais ou menos diversos, e com a intenção de se referir a situações específicas. Assim, a junção dos termos "media" e "polis" na mesma palavra tem possibilitado interessantes discussões na arquitetura, nos estudos das comunicações, na sociologia e nas ciências políticas, e na política público-governamental. Nas palavras de Rodger, Barnett e Cochrane (2008):

Why 'mediapolis'? In using this term, our intention was not to propose a groundbreaking new concept. Indeed, the initial rationale was significantly less ambitious. Mediapolis was just a way of provocatively interweaving media, politics and cities, by joining together 'media' with the various urban and political meanings of 'polis' (e.g. city, state, citizens). It pointed out, rather nicely and in a single word, an opening for discussion on the intersections of media with cities and urban spaces as settings for, and objects of, politics.

$\mathrm{Na}$ medida em que o termo "media" evoca a tecnologia, a informação, as comunicações, a imprensa, a publicidade, a propaganda, as interações mediadas por computadores, a internet, a indústria cultural, as artes e as estéticas midiáticas, há um largo espectro de possibilidades para pensar a mediapolis. Por outro lado, o termo "polis" remete à cidade, ao Estado moderno, à cidadania, à democracia, à mobilização, ao engajamento e à participação na política. Por exemplo:

The use of NCT (new communication technology) to create urban utopias. (...) Metropolitan form is being affected by the NCT and the computer linked to eletronic communication creates information machines which "may change the shape of our cities. (...) The decline of the traditional print city of mass media and the death of centrality are concurrent with the multiple centers and many mediums of what might be called the "mediapolis" of the post-industrial city and society (Burd, 1982). 
Para Burd, as novas tecnologias de comunicação iriam redesenhar o traçado físico tradicional das cidades - que possuíam um centro povoado e ativo. A nova cidade, filha do impacto tecnológico, é denominada mediapolis: uma cidade virtual com atividade dispersa - na economia, na imprensa e na demografia. Para o autor, ter múltiplos centros e mídias poderia afetar negativamente a coesão entre os cidadãos, portanto a cidadania. A relação entre tecnologia e traçado das cidades também aparece em Seligman:

Artpolis revolutionized public architecture in Japan (...) Artpolis represents a comprehensive effort to produce inventive public projects, publicize architecture, and foster public spheres through architecture. (Creating) programs to cultivate broad public engagement (...). (Seligman, 2013a)

Shiroishi's Mediapolis (1992-2004) created a city scale variation of the Artpolis project enterprise guided by a notion of urban acupuncture. (...) With the Mediapolis program they aimed: 1) to discover and develop Shiroishi's regional individuality, while seeking the highest standard of living in Japan; 2) to protect historical urban structures (...); 3) to develop the city through "self-healing" acupuncture strategies that stimulate points across the city. (...) Horiike expressed intentions to use the power of media to build the city. (Seligman, 2013b)

Aqui Mediapolis implicaria um novo paradigma para a arquitetura pública: através da noção de acupuntura urbana, a ideia seria promover a auto-cura das cidades ao estimular certos espaços. Buscava-se melhor qualidade de vida, fomentar o engajamento cívico e, ao mesmo tempo, desenvolver a esfera pública local. As locações estratégicas iriam oferecer infraestrutura e treinamento em TI (tecnologia da informação) para a comunidade, no formato de centros de multimídia. Esses "Hubs" de tecnologia também vão aparecer num projeto de Singapura:

Located in one-north, the 19-hectare Mediapolis was launched in December last year. It is set to become a self-contained media ecosystem comprising soundstages with green screen capabilities, digital production and broadcast facilities and media schools. There will also be centres for activities in interactive digital media and research and development; computer-generated imagery and visual effects; games and animation; and intellectual property creation and digital 


\section{míDiA
eCOti
DiAno}

rights management. Mediapolis was created in response to Singapore's expanding media sector. (...) Global media giants such as Lucasfilm, Linden Lab, EA, Ubisoft and Rainbow SpA have also set foot here. And more media activities such as the upcoming shoot of Jan de Bont's Point Break 2 will take place this year. The government therefore came up with Mediapolis to position Singapore as a media hub (Business Times, 2009).

Portanto, mediapolis é entendida tanto como a virtualização da cidade física tradicional (cujo impacto redesenharia o traçado da cidade real), quanto como intervenções arquitetônicas conscientes (ora como a construção de centros multimídia numa acupuntura urbana, ora como a construção de hubs multimídia no formato de parques industriais). Em todos estes casos, a mídia e a arquitetura caminham juntas para remodelar o espaço da cidade - e, ao mesmo tempo, remodelar os espaços de convivência e de cooperação entre as pessoas. Esta nova cidade tem vários nomes, dentre eles 'mediapolis':

Some call it the 'meta-city', others the 'network city', the 'media city', the 'software sorted city' or the 'mediapolis'. What all these catchwords have in common is a description of the 21 st century city as a double entity. The physical, geographical city with its piazza's, its neighborhoods and highway interchanges is overlaid with the 'Hertzian space' of electronic communication-, information- and observationnetworks (...). Concurrently, the domain of digital space is increasingly becoming physical, now that RFID-chips turn objects into 'spimes' and 'blogjects', creating an "internet of things". These networks and media are not merely tools, used by urbanites for more efficient, safe, convenient, or exclusive lifestyles. Their proliferation might change our ideas of time, space, identity, belonging, solidarity and citizenship; they will have an effect on the broader issue of 'urban culture' and the city itself. (...) Urban space has thus become 'hybrid space', 'a conceptual space created by the merging of borders between physical and digital spaces'. (Waal e Lange, 2010)

Living in Supermodernity (Auge, 1995), 'Mediapolis' is a term coined to describe the dual entities of the twentyfirst century city: the physical place and the superimposed virtual informatics networks. (...) Hybrid Space' // 'Augmented Space' // 'The Interactive City'/ 'Mediapolis': the physical environment has been embedded with information, 
verlayed with the aid of technologies and mobile media which incorporate Wifi, GPS etc. The topography of the contemporary urban space and consequently our perception of it, is constantly being recofigured and has thus become interactive. This 'hybrid space', augmented reality is a mesh between the two spaces. The user attempts to "overlay physical objects with virtual objects in real-time (and) allowing people to experience the virtual as if it were real." (Christofidou, 2012)

Mediapolis é, então, a nova cidade, o novo cidadão, a nova cidadania: espaço real e virtual, permeado de homens e máquinas, que interagem e comunicam para gestar e gerir a nova vida em comum. Este espaço híbrido (analógico e digital), com agentes híbridos (de carbono e silício), oferece novas formas de viver e agir em comunidade. Disso decorre, igualmente, uma nova cultura - que representa o espaço urbano (Jong e Schuilenburg, 2006) e está saturada de mídia e informação (Inkinen, 2004).

We use the term 'pop philosophy' to refer to the approach that uses popular media as material to analyse the contours of our everyday environment. This philosophy opens a playful space in the margins of the primarily academically tinted discourse of the city. In Mediapolis, this takes place by investigating the representation of urban space in pop culture. What does urban reality look like in those virtual and sonic environments and how does this affect our physical reality? (...) The basic idea of Mediapolis is that the popular media environments, in which technical, cultural and economic changes join in a unique way, offer an opportunity to approach our living environment from a different angle. (Jong e Schuilenburg, 2006)

Mediapolis is a critical, multi-disciplinary anthology that discusses, e.g., the theories, problems, and possibilities of multimedial communication, computer interaction, hypertextual representation of knowledge, contemporary utopias, television broadcasting, semiotics of media, and sexuality in the cyber age. Instead of technological determinism or trendy techno-optimistic rhetoric, the book focuses on an analytical approach to contemporary media, future technologies, and electronic texts. In the mentioned anthology, I use the title "Mediapolis" (cf. Neil Postman's Technopoly [1993] and Nigel Calder's Technopolis [1969]) to describe the information and media saturated culture of our time; the possibilities and problems it brings. (Inkinen, 2004)

A política japonesa de investir na construção de centros multimídia para materializar uma esfera pública propícia ao engajamento cívico encontra, no conceito de 
Mediapolis desenvolvido por Silvestone (2006), interessantes convergências e divergências. A despeito de o termo 'mediapolis' ser utilizado por vários autores, como os supra citados, o livro de Roger Silvestone é talvez a obra mais citada sobre o assunto, e é preciso checar em que medida suas definições diferem das dos autores abordados até o momento.

\section{A Mediapolis de Silvestone}

Até agora a mediapolis foi definida através de dois aspectos: de um lado, uma noção arquitetônica; de outro lado, uma moção (i.e. uma evolução) tecnológica. Ou seja, de como o avanço das novas tecnologias de informação e comunicação tem reconfigurado os espaços físicos e virtuais, e, por consequência, de como essa reconfiguração tem afetado as vivências e convivências em sociedade. Roger Silverstone aborda tais problemas, por certo, mas enfatiza também as questões políticas e morais. Em seu livro, o autor procura definir mediapolis como:

[T]he mediated public space where contemporary political life increasingly finds its place, both at national and global levels, and where the materiality of the world is constructed through (principally) electronically communicated public speech and action. (...) I therefore propose mediapolis, in the singular, notwithstanding the manifest fragmentation of the delivery platforms, channels and cultures of global media. (...) The mediapolis is, I intend, the mediated space of appearance (...). It is through communications conducted through the mediapolis that we are constructed as human (or not), and it is through the mediapolis that public and political life increasingly comes to emerge at all levels of the body politic (or not). (Silverstone, 2006)

Assim, a noção de arquitetura evolui para o conceito de espaço desterritorializado, em que a concepção de cidade evapora e é substituída pela de comunidade transcendente; enquanto a moção tecnológica vai redefinir a ação (falar e fazer) política - já que mudam as arenas, as formas de agir e interagir, e as implicações de cada performance. Para ele, "the mediapolis is a moral space, a space of hospitality, 


\section{eco DiAno}

responsibility, obligation and judgement", e isso redireciona os conceitos de "justiça" e "responsabilidade" para o primeiro plano da discussão.

Silverstone moves on to unfold some of the criteria of media hospitality, media justice and media ethics as morally based reference points which can contribute to achieve a fully effective communication in mediapolis. It is a communication practice which he further argues is based on: a) A mutuality of responsibility between producer and receiver; b) A degree of reflexivity by all participants in the communication and; c) A recognition of cultural difference. In other words, Silverstone regards this mediated public sphere, 'mediapolis' is ideally a dialogic space which 'is both an encompassing global possibility and an expression of the world's empirical diversity’. (Cogo, ElHajji e Huertas, 2012)

The moral argument (of Mediapolis). Media put us in contact with global others; this evokes moral responsabilities. (...) Moral demand for reflexivity, recognition of cultural difference. (...) Moral response via thinking, speaking, listening; and acting (...). (Dalghren, 2013)

Sai-se propriamente da esfera urbana para a esfera moral, troca-se efetivamente de interesse sobre o impacto que a tecnologia possa ter: antes, era sobre a política de comunicação (quem usa qual mídia e como); agora, é sobre a comunicação política (o impacto sobre a vida comunitária do uso das mídias). Migra-se dos usos das mídias para os usuários das mídias, das considerações tecnológicas para as filosóficas, do reino da técnica para o da estética. A diversidade humana passa a ser encarada como fundamento ético e moral das relações, permeadas pelo mútuo reconhecimento e responsabilidade na convivência. De certo modo, o ser humano é a nova cidade (o novo ambiente), e sua arquitetura interna (seu vigamento ético e moral) é que responde à tecnologia (menos dos objetos físicos e mais dos processos sociais).

\section{Parte II}

\section{Comunicadores Artificiais}

Para alguns autores (Fogg, 1998), as máquinas não são apenas ferramentas ou mídias; as máquinas podem ser também atores sociais. Se estudos sobre comunicação homem-máquina não são exatamente uma novidade, a diferença é que, atualmente, percebe-se uma preocupação maior com a modelagem e a programação da 


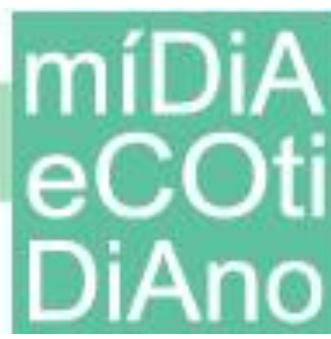

comunicação, e, mais especialmente, com a criação de comunicadores artificiais. Há estudos empíricos e teóricos que lidam especificamente com o tratamento computacional da persuasão - entendida como um tipo de comunicação (Guerini, 2003). Naquilo que temos chamado de comunicação artificial, há artigos com experimentos computacionais para simular o surgimento e a evolução da comunicação (Lipson, 2007), e a utilização de autores e teorias pertencentes à história das "Ciências das Comunicações" para modelagem e programações computacionais. Em resumo, nunca a comunicação esteve tão próxima de um tratamento automático - bem verdade que num enfoque multimodal, e não exclusivamente linguístico.

\section{Agentes Artificiais Sociáveis}

Uma série de pesquisas e projetos originados nos departamentos de computação tem oferecido significativos avanços na área de agentes artificiais: sejam virtuais ou reais, possuam deliberadamente maior ou menor semelhança com o aspecto humano, proponham uma ou mais modalidades de expressão (gestual, linguístico, etc.). Dentre as muitas novidades num campo repleto de robôs e androides, ressaltam-se as pesquisas sobre a modelagem e programação de sociabilidade em máquinas (Breazeal, 1999). Afinal, um comunicador artificial tem que ser, por definição e previamente, um ator social - no sentido de que não existe comunicação no solipsismo.

O objetivo é chegar a agentes artificiais capazes de interagir o mais naturalmente possível com humanos, estejam eles a sós ou em grupo. Esse agente social artificial deve incluir "tecnologias de interação que podem assumir o papel de parceria social, e ter capacidade comunicativa para processar-gerar signos verbais e não-verbais (e.g. gestual)" (Mehler, 2008). Ou seja, os pesquisadores têm se dedicado a desenvolver sistemas computacionais que sejam mais que ferramentas ou mídias; que atuem efetivamente atores sociais. 


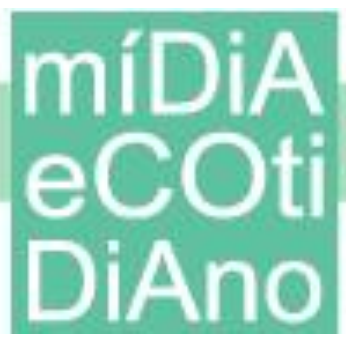

\section{Web Social}

Spivack (2006), abordando a terceira geração da web, apresenta uma importante comparação. Para ele, teríamos: a) a Web 1.0 - a primeira geração, onde o foco era no acesso e na comercialização; b) a Web 2.0 - a segunda geração, com ênfase nas redes sociais e ferramentas de comunicação, e cujas palavras centrais são compartilhamento e colaboração; e c) a Web 3.0 - a terceira geração, chamada de "a rede inteligente", povoada por máquinas artificialmente inteligentes e capazes de aprendizagem, e de "rede semântica", por causa de um novo formato de registro de seu conteúdo. A Web 2.0 é a que vem sendo chamada propriamente de "web social", e a Web 3.0 ainda está a meio caminho de efetivar-se. Por exemplo, a reescrita semântica da web permitiria que máquinas lessem, entendessem, julgassem e agissem autonomamente enquanto usuários da internet: ou seja, na Web 3.0 as próprias máquinas se tornariam usuários inteligentes da internet, agindo em nome e para benefício dos usuários humanos - elas uma espécie de procuradores na navegação.

A inteligência em máquinas sociais incluiria, nas palavras de Nardi (2002), os seguintes atributos : a) comunicação: o agente pode obter, capturar, lembrar, inferir, testar, e checar informação socialmente relevante, tais como o nome, idade e trabalho da pessoa, sentimentos sobre si e sobre outros, valores, etc. incluindo informação de "processo" como o estilo de interação-social da pessoa.; b) afiliação social: o agente é capaz de intercambiar informação entre ou de ligar pessoas diferentes que constituem sua rede social, pode checar o valor social do que aprendeu, falar de terceiros, determinar o comportamento apropriado ao seu círculo social, tirar vantagens de oportunidades, etc.; c) Auto-acesso: o agente pode examinar, monitorar, mudar, atualizar, e avaliar sua própria programação enquanto interage, e pode engajar-se numa conversa consigo mesmo para se entender e obter auto-estima, nos mesmos termos que faz com outra pessoa; d) Contexto: o agente inteligente comunica em tempo real com mais de uma pessoa, fazendo escolhas contextualmente dirigidas. Basta imaginar esses agentes sociais artificiais da Web 3.0 navegando, como usuários inteligentes e autônomos, pela Web 2.0. Essa ideia permite conceber pessoas e máquinas trabalhando 


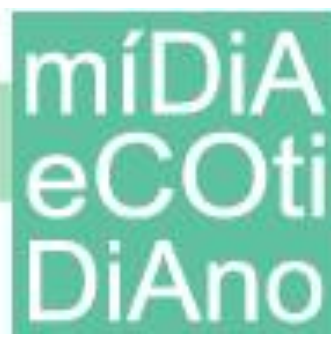

juntas, sociabilizando e cooperando, compartilhando informações e comunicando em rede.

\section{Agentes Artificiais e Web Social}

Imbres (2008) associa a Web 3.0 a robôs conversacionais, capazes de se comunicarem com as pessoas e de assumirem a posição de "connection brokers". O autor chega a expressar a possibilidade de que agentes comunicativos artificiais possam assumir as atribuições de um(a) relações públicas. O Design Incubation Centre (2008), em seu Workshop Robô 2.0, busca formas de integrar robôs humanizados no dia a dia das pessoas, inclusive na Web 2.0; onde, em suas palavras, "vemos a emergência de um novo ecossistema social". A convergência entre agentes comunicativos artificiais da Web 3.0 e as redes sociais da Web 2.0, mantendo em mente toda a pesquisa em modelagem e programação de sociabilidade artificial, leva a discussão a um novo patamar. Neste contexto de comunicadores artificiais, capazes de argumentar e debater inteligentemente com humanos, e mesmo de eventualmente persuadi-los, é que importa discutir o futuro daquilo que, hoje, se chama Web Social (Coutinho e Junior, 2007).

Atualmente, sistemas inteligentes on-line são capazes de emular um ou vários atores em redes sociais, interagindo e participando em fóruns, atuando colaborativamente na construção e oferta de conteúdo, enfim, fazendo parte da tão propalada inteligência coletiva (Silva, 2007). A tendência é que, à medida que avança a pesquisa em agentes artificiais comunicativos e em sociabilização de agentes artificiais, e dado o caráter mediador da internet, há de ficar mais urgente a necessidade de considerar a participação de humanoides (Zhao, 2006) em ambientes como a Web Social. Embora a pesquisa de modelagem e programação em uma gama considerável de fatores humanos tenha muito a avançar (caso da confiança), não é cedo para pensar em redes sociais colaborativas onde o computador seja mais que ferramenta e mídia, e passe a ser também ator social. 


\section{míDiA

Parte III

\section{Mediapolis e Agentes Artificiais}

Pensar a mediapolis no contexto de agentes artificiais é um desafio necessário. Não se trata exatamente de discutir a viabilidade técnica de obter máquinas "realmente autônomas e inteligentes" (dado que autonomia e inteligência são conceitos espinhosos quando aplicados a máquinas), mas, antes, de observar o impacto do atual estágio tecnológico sobre a cidade e os cidadãos.

A vida como a entendemos está cada vez mais permeada por objetos tecnológicos (e.g. smartphones), num sentido em que não se admite a possibilidade de viver sem acesso à internet: em si, um mundo paralelo. Se à luz de Silverstone o ser humano é que é a nova cidade, importando seu vigamento moral e ético, vale a pena perguntar: o que seria a mediapolis hoje?

A palavra "hibridização" oferece uma conveniente solução, pois não é mais possível pensar separadamente em humano e máquina, real e virtual, analógico e digital, individual e social, privado e público, hardware e software, orgânico e inorgânico etc. Outra chave é considerar a mútua convergência entre os extremos de tais conceitos, por exemplo: ao mesmo tempo em que se trabalha para humanizar as máquinas em todos os seus aspectos, os seres humanos também parecem apresentar comportamentos crescentemente "robóticos".

Assim, a evolução da mediapolis sugere um próximo estágio relativamente complexo: a cidade deixa de ser um espaço ou ambiente (não importa se real ou virtual), e perde igualmente algo de sua humanidade. Por um lado, a cidade passa a ser um sentimento de existência e de pertença, uma multiplicidade de sensações que migram constantemente, comunidades que emergem e desaparecem ao sabor do momento. Por outro lado, a cidade celebra um Coup d'État dos objetos, com tijolos evoluindo para smartphones e 'construções' adquirindo interatividade e habilidades sociais.

À medida que avançam os esforços em programar um comportamento ético em máquinas (www.roboethics.org), que vivemos mais e mais através das máquinas (Facebook e SecondLife), e que a 'internet das coisas' tem chegado a prédios e 


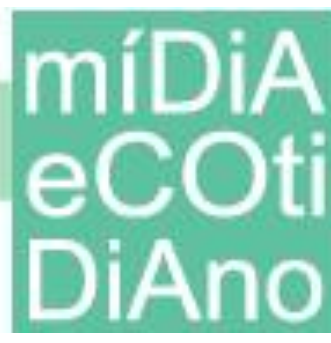

utensílios domésticos e carros, cabe repensar conceitos como ética e moral - antes exclusivos aos seres humanos. Se máquinas são também atores sociais (e não apenas mídias), a mediapolis de Silverstone vai necessitar de ajustes; quem sabe sendo rebatizada como imediapolis - com o prefixo i a evocar intelligent.

Assim, a imediapolis lança o desafio de conceber a democracia e a cidadania estendidas às máquinas, cujo estatuto jurídico já tem sido debatido (Darling, 2012); e de, com maior estranhamento talvez, conceber a extensão das máquinas nos seres humanos, o que o advento dos cyborgs já inaugurou (Reddy, Paul, Jyothi, 2013). Essa hibridização do silício no carbono (e vice-versa), no esforço por transformar seres humanos em mídias e mídias em seres humanos, subverte inadvertidamente qualquer noção de cidade.

O escopo deste artigo é reconhecer esta mudança já em curso, sem necessariamente defini-la ou descrevê-la - pois que ainda inacabada. A introdução de mídias inteligentes na mediapolis, mais corretamente nomeados por agentes sociais artificiais (visto serem mais que mídias), conduziu à sugestão do conceito ligeiramente modificado de imediapolis; que, por sua vez, há de lançar nova luz sobre o trabalho de Silverstone e demais autores.

\section{Conclusão}

As diversas camadas geológicas do conceito de mediapolis podem ser resumidas assim: 1) viés arquitetônico; 2) viés tecnológico; 3) viés comunicacional; 4) viés sociológico e político; e 5) viés filosófico. Claro, cada uma dessas camadas interpenetra as demais, sem jamais delas se separarem. Assim, a trajetória (não exatamente linear) percorrida ilustra como a mediapolis pode ser, ao mesmo tempo, a cidade real, a cidade virtual, a infraestrutura de mídia, os processos de comunicação que são a causa ou o efeito das mídias, a novas vivências sociais e ações políticas derivadas das tecnologias, e as novas éticas e a nova moral aí gestadas. 
A maior contribuição de Silvestone (2006) foi, sem dúvidas, oferecer um contraponto a Habermas, propondo a mediapolis, antes, como um "chaotic, cacophonic space of global media" que "resides beyond logic, rationality; (with) efficacy always uncertain" (Dalghren, 2013). Essa chacoalhada nas certezas, no controle e na previsão Habermasianas pavimentaram, queremos crer, o caminho que conduziu à discussão da ética e da moral associadas às mídias. $\mathrm{O}$ que nos possibilitou, a seu turno, afirmar que, afinal, o homem é que é a nova mediapolis - e o que importa é sua arquitetura ética e moral.

Todavia, a introdução de mídias autônomas e inteligentes, capazes de sociabilização com humanos dentro e fora da internet, significou um passo além, quiçá exigindo um novo termo para um novo conceito: a imediapolis. Esta realidade repleta de homens-máquinas e máquinas-humanas tornou a tarefa de pensar a cidade algo difícil: se os próprios edifícios se tornam inteligentes e, eventualmente, atores sociais capazes de interação, é a noção de espaço e ambiente que estão em crise.

Mas, ainda que seres e coisas possuam status de agentes sociais, e que as 'construções' da cidade (prédios, carros, objetos) tenham deixado de ser apenas locais no espaço, lançando-se ativamente para interagir inteligentemente com as pessoas, resta ainda por desenvolver mais apropriadamente o conceito proposto de imediapolis - aqui simplesmente esboçado pela primeira vez. A introdução de novos agentes sociais artificiais é a novidade na/da polis, visto que constituem, em parte, a própria cidade em sua materialidade. Logo, é a cidade que se eleva a mídia, em meio a mídias que se elevam a agentes sociais. Enfim, é a própria cidade a tornar-se um ator social.

\section{Referências Bibliográficas}

BREAZEAL, Cynthia; Scassellati, Brian (1999) How to Build Robots that Make Friends and Influence People. In: IROS99. Kyonjiu, Korea.

BUSINESS TIMES. (2009) Creative Buzz at Mediapolis. March. Acesso em 10 de Julho de 2014: https://1 north.wordpress.com/2009/04/06/creative-buzz-at-mediapolis/

BURD, Gene. (1982) Urban Uses and Social Impact of New Communication Technology: a critical and philisophical perspective. Qualitative Studies Division, University of Texas, July. 


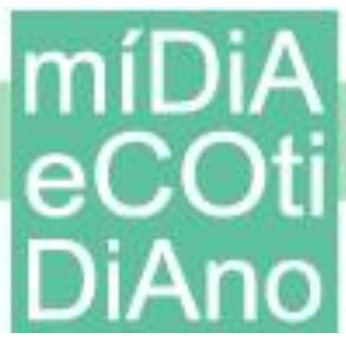

CHRISTOFIDOU, Eirini. (2012) Mediapolis - The convergence of the physical and digital spaces in the urban realm and locative media technologies, Master Dissertation, University of Sheffield.

COGO, Denise; ElHajji, MOHAMMED; Huertas, AMPARO (eds.) (2012) Diaspora, migration, communication technologies and transnational identities. Bellaterra, Institut de la Comunicació, Universitat Autònoma de Barcelona.

COUTINHO, C.P.; Junior, J.B.B. (2007) Blog e Wiki: os futuros professores e as ferramentas da Web 2.0. In: SIIE.

DALGHREN, Peter. (2013) Cosmopolitanism and Global Citizenship: the Rhetoric of Moral Agency. University of Copenhagen, Jan. 15-18.

DARLING, Kate. (2012) Extending Legal Rights to Social Robots. In: We Robot Conference, April, University of Miami, Miami, USA.

DESIGN INCUBATION CENTRE (2008) Robots 2.0 Workshop. Disponível: http://www.designincubationcentre.com/index/past-workshops/robots-20/index.html

FOGG, Brian J. (1998) Persuasive Computers: Perspectives and research directions. In: Proceedings of the CH198. Conference of the ACM/SIGCHI. New York: ACM Press.

GUERINI, Marco et alli (2003) Persuasion Models for Intelligent Interfaces. In: Proceedings of the IJCAI Workshop on Computational Models of Natural Argument. Acapulco, Mexico.

IMBRES, Peter (2007) Web 3.0: Attack of the Conversation Robots. Disponível: http://point-oh.com/?p=119

INKINEN, Sam. (2004) On the Electronic Zeitgeist. Aspects of the Information Society, Homo Symbolicus and the Global Village. European Institute for a Sustainable Information Society (EISIS), Helsinki, Finland.

JONG, Alex de; Schuilenburg, Marc. (2006) Mediapolis - popular culture and the city. Rotterdam: 010 Publishers, 2006.

LIPSON, Hod (2007) Evolutionary Robotics: Emergence of Communication. In: Current Biology. Vol. 17, No 9.

MEHLER, Alexandre et alii (2008) Handbook of Technical Communication. Berlin/New York, Mouton de Gruyter. Disponível: http://valian.kgf.unifrankfurt.de/hal8/hal8.pdf

NARDI, Dario (2002) Intelligence as Communication-Roles and Needs of Socially Embedded Agents. In: Proceedings of the Lake Arrowhead Conference on Computational Social Science and Social Complexity. 
REDDY, T. R.; Paul, S.; Jyothi, B. V. (2013) The Rise of The Cyborgian Epoch. In: International Journal of Computer and Information Technology, 2(2).

RODGERS, Scott; BARNETT, Clive; COCHRANE, Allan. (2008) Mediapolis - an introduction. In: Mediapolis - media practices and the political spaces of cities. Interdisciplinary workshop. The Open University, Milton Keynes, UK, 9-10 June, pp. 310 .

SELIGMANN, Ari. (2013a) Artpolis Legacies Proliferation of Public Architecture Programs for Urban Regeneration in Turn of the Century Japan. In: Brown, Alexandra; Leach, Andrew (ed.). 30th Annual Conference of the Society of Architectural Historians, Australia and New Zealand. Gold Coast, Queensland, Australia, July 2-5, pp. 83-95.

SELIGMANN, Ari. (2013b) Revitalizing Cities: Architecture as Regional Catalyst from Bubble Era Blowouts to Fukushima Fallouts and Beyond. Japanese Studies Association of Australia, Conference, Japan Institute, ANU College of Asia \& the Pacific, The Australian National University, Canberra, Australia.

SILVA, Crystian K.P. (2007) Web 2.0: a migração para a Web Social. Ciência da Computação, UFJF.

SILVERSTONE, Roger. (2006) Media and Morality: On the Rise of the Mediapolis. London School of Economics and Political Science, London, UK.

SPIVACK, Nova (2006) The Third-Generation Web is Coming. Conceito disponível: http://www.kurzweilai.net/meme/frame.html?main=/articles/art0689.html

TUFTE, Thomas. (2011) Mediapolis, Human (In)Security and Citizenship. Communication and Glocal Development Challenges in the Digital Era. In: Christensen, Miyase; Jansson, Andre; Christensen, Christian (ed.). Online Territories: Globalization, Mediated Practice and Social Space. New York: Peter Lang, pp. 113-131.

WAAL, Martijn de; LANGE, Michiel de. (2010) The Mobile City: a conference on locative media, urban culture and identity. Netherlands Architecture Institute, Rotterdam, February 27ᄀ-28.

ZHAO, Shanyang (2006) Humanoid Social Robots as a Medium of Communication. In: New Media \& Society, Vol. 8, No. 3, SAGE. 